\title{
Stability of solutions for generalized fractional differential problems by applying significant inequality estimates
}

\author{
Mohammed D. Kassim', Thabet Abdeljawad ${ }^{2,3,4^{*}}$, Saeed M. Ali ${ }^{1}$ and Mohammed S. Abdo ${ }^{5 *}$ (D)
}

"Correspondence:

tabdeljawad@psu.edu.sa; msabdo1977@hoduniv.net.ye

2Department of Mathematics and General Sciences, Prince Sultan University, Riyadh, Saudi Arabia ${ }^{5}$ Department of Mathematics, Hodeidah University, Al-Hodeidah, Yemen

Full list of author information is available at the end of the article

\section{Springer}

\begin{abstract}
In this research paper, we intend to study the stability of solutions of some nonlinear initial value fractional differential problems. These equations are studied within the generalized fractional derivative of various orders. In order to study the solutions' decay to zero as a power function, we establish sufficient conditions on the nonlinear terms. To this end, some versions of inequalities are combined and generalized via the so-called Bihari inequality. Moreover, we employ some properties of the generalized fractional derivative and appropriate regularization techniques. Finally, the paper involves examples to affirm the validity of the results.
\end{abstract}

MSC: 26A33; 34A08; 34E10

Keywords: Asymptotic behavior; Boundedness; Generalized fractional derivative; Fractional differential equation

\section{Introduction}

Fractional calculus has gotten much consideration from analysts and engineers, as well as a tool in various areas of engineering, applied mathematics, and physics. Fractionalorder differential equations are used to study plentiful phenomena such as fluid mechanics, plasma physics, optical fibers, nonlinear oscillations of an earthquake, flow in nonlinear electric circuits, biology, aerodynamics, mechanics, and regular variations in thermodynamics. Actually, the transform from theoretical to the application aspect of fractional calculus was appeared due to the work by Bagley and Torvik [1-3]. A generalized fractional derivative with respect to function $t^{\rho}$ is a novel sort of fractional derivatives, which has been presented by Kilbas et al. [4], and then modified by Katugampola [5] and Almeida et al. [6]. In a series of papers [7-18] the authors studied the qualitative analysis for some classes of fractional differential equations involving this generalized fractional derivative.

Fractional-order derivatives can describe the asymptotic behavior of some nonlinear systems more comprehensively as compared to integer order. Further, integer-order derivatives are the special case of fractional-order derivative. The most significant peculiarity of the considered derivative here is that it provides a general platform that covers most classical fractional derivatives (e.g. Riemann-Liouville and Hadamard). That is why

(c) The Author(s) 2021. This article is licensed under a Creative Commons Attribution 4.0 International License, which permits use sharing, adaptation, distribution and reproduction in any medium or format, as long as you give appropriate credit to the original author(s) and the source, provide a link to the Creative Commons licence, and indicate if changes were made. The images or other third party material in this article are included in the article's Creative Commons licence, unless indicated otherwise in a credit line to the material. If material is not included in the article's Creative Commons licence and your intended use is not permitted by statutory regulation or exceeds the permitted use, you will need to obtain permission directly from the copyright holder. To view a copy of this licence, visit http://creativecommons.org/licenses/by/4.0/. 
this fractional derivative is often referred to as a generalized fractional derivative. The said operator has been used in many articles. The said operator has been found useful in dealing with many real problems.

Fractional differential problems can describe the dynamics of several complex and nonlocal systems with memory. They emerge in numerous scientific and engineering areas. Especially, nonlinear systems describing various phenomena can be modeled with fractional derivatives. These fractional operators possess memory and this memory is efficient in describing and modeling complex systems nonlocally.

In this regard, the researchers studied many models and used a fractional-order derivative to describe the solution of them. Among them studying the asymptotic behavior of such models has meaningful interpretations like permanence, instability, and chaotic developments. The main aim of this article is to investigate the long-time behavior of solutions of the following problem:

$$
\left\{\begin{array}{l}
\rho \mathfrak{D}_{a}^{\sigma} \varpi(\varkappa)=\mathfrak{F}\left(\varkappa, \varpi(\varkappa),{ }^{\rho} \mathfrak{D}_{a}^{\gamma} \varpi(\varkappa)\right), \quad 0<\gamma<\sigma<1, \rho>0, \varkappa>a>0, \\
\left.{ }^{\rho} \mathfrak{I}_{a}^{1-\sigma} \varpi(\varkappa)\right|_{\varkappa=a}=b \in \mathbb{R},
\end{array}\right.
$$

here ${ }^{\rho} \mathfrak{I}_{a}^{r}$ and ${ }^{\rho} \mathfrak{D}_{a}^{r}$ are the generalized fractional integral and derivative of order $r>0(r \in$ $\{\sigma, \gamma, 1-\sigma\})$, respectively. These types of operators are defined in the next sections. In [19], Furati and Tatar studied the problem

$$
\left\{\begin{array}{l}
\mathfrak{D}_{a}^{\vartheta_{1}} \varpi(\varkappa)=\mathfrak{F}(\varkappa, \varpi), \quad 0<\vartheta_{1}<1, \varkappa>0, \\
\left.\varkappa^{\left(1-\vartheta_{1}\right)} \varpi(\varkappa)\right|_{\varkappa=0}=b \in \mathbb{R},
\end{array}\right.
$$

and they proved that the solutions decay to zero. In this respect, Furati et al. [20] considered the problem

$$
\left\{\begin{array}{l}
\mathfrak{D}_{0}^{\vartheta_{1}, \vartheta_{2}} \varpi(\varkappa)=\mathfrak{F}(\varkappa, \varpi), \quad 0<\vartheta_{1}<1, \varkappa>0, \\
\left.\varkappa^{\left(1-\vartheta_{1}\right)\left(1-\vartheta_{2}\right)} \varpi(\varkappa)\right|_{\varkappa=0}=b \in \mathbb{R}
\end{array}\right.
$$

here $\mathfrak{D}_{0}^{\vartheta_{1}, \vartheta_{2}}=\mathfrak{I}_{0}^{\vartheta_{2}\left(1-\vartheta_{1}\right)} D \mathfrak{I}_{0}^{\left(1-\vartheta_{2}\right)\left(1-\vartheta_{2}\right)}$. They demonstrated that solutions of this problem decay as a power function. Plociniczak [21] studied the equation

$$
{ }^{c} \mathfrak{D}^{\vartheta_{1}} \varpi(\varkappa)=\lambda q(\varkappa) \varpi(\varkappa), \quad 0<\vartheta_{1}<1, \varkappa>0,
$$

where ${ }^{c} \mathfrak{D}^{\vartheta_{1}}$ is the Caputo derivative and $q(\varkappa) \sim C_{q} \varkappa^{v}>0, v>0$. The author showed that the solutions of (1.4) obey the asymptotic properties according to values of $\lambda$. Medved et al. [22] considered the problem

$$
{ }^{c} \mathfrak{D}_{0}^{\vartheta_{1}} \varpi(\varkappa)=\mathfrak{F}\left(\varkappa, \varpi(\varkappa),{ }^{c} \mathfrak{D}_{0}^{\vartheta_{2}} \varpi(\varkappa)\right), \quad 0<\vartheta_{2}<\vartheta_{1}<1, \varkappa>0 .
$$

They demonstrated that any solution of (1.5) has the asymptotic property $\varpi(\varkappa)=c \varkappa^{\vartheta_{2}}+$ $o\left(\varkappa^{\vartheta_{2}}\right)$ when $\varkappa \rightarrow \infty$ for some $c \in \mathbb{R}$. Kassim et al. [23] studied the equation

$$
\mathfrak{D}_{0}^{\vartheta_{1}} \varpi(\varkappa)=\mathfrak{F}\left(\varkappa, \varpi(\varkappa), \mathfrak{D}_{0}^{\vartheta_{2}} \varpi(\varkappa)\right), \quad 0 \leq \vartheta_{2}<\vartheta_{1}<1, \varkappa>0
$$


They proved that solutions of (1.6) decay to zero. Recently, Kassim et al. [24] investigated the equation

$$
\mathcal{D}_{a}^{\vartheta_{1}} \varpi(\varkappa)=\mathfrak{F}\left(\varkappa, \varpi(\varkappa), \mathcal{D}_{a}^{\vartheta_{2}} \varpi(\varkappa)\right), \quad 0 \leq \vartheta_{2}<\vartheta_{1}<1, \varkappa>a,
$$

where $\mathcal{D}_{a}^{\kappa}$ is the Hadamard fractional derivative of order $\kappa \in\left\{\vartheta_{1}, \vartheta_{2}\right\}$ and they showed that solutions of (1.7) decay to zero.

Moreover, in 2020, the same authors [25] discussed the equation

$$
{ }^{c} \mathfrak{D}_{0}^{\vartheta_{1}} \varpi(\varkappa)=\mathfrak{F}\left(t, \varpi(\varkappa),{ }^{c} \mathfrak{D}_{0}^{\vartheta_{2}} \varpi(\varkappa)\right), \quad \vartheta_{2}<\vartheta_{1} .
$$

They proved that the solutions of (1.8) approach power type functions for $1<\vartheta_{1}<2$ and bounded for $0<\vartheta_{2}<1$. For more results related to asymptotic behavior, we refer to [19, $20,23,26-32]$, and the references therein. In this letter, generalization of the results in $[19,20,22-24]$ to problem (1.1) was established. In particular, the problems (1.6) and (1.7) studied in [23,24] become special cases of (1.1) when $\rho=1$ and $\rho \rightarrow 0$, respectively.

In the present work, we prove under certain conditions the solutions of (1.1) decay towards zero as $\left(\frac{\varkappa^{\rho}-a^{\rho}}{\rho}\right)^{\vartheta_{1}-1}$. The investigated fractional derivatives belong to a general class of fractional operators including Riemann-Liouville, Caputo, and in the limiting case to zero the Hadamard and Caputo-Hadamard fractional derivatives. The used techniques are novel and new and applied in a clever way. The studied fractional equation is studied in this category for the first time and this will help researchers to proceed more in this direction.

In Sect. 2, we prepare some materials. In Sect. 3, we state and prove the main result. Illustrative examples are given in Sect. 4. Concluding remarks are discussed in the last section.

\section{Preliminaries}

In this section, we briefly recall some definitions, lemmas, properties and notations and well-known estimations we will use later.

Definition 2.1 ([12]) Let $\rho>0$ and $0 \leq \gamma<1$, we introduce the spaces $C_{\rho, \gamma}[a, b]$ and $C_{\rho, \gamma}^{n}[a, b]$ as follows:

$$
\begin{aligned}
& C_{\rho, \gamma}[a, b]=\left\{g(\varkappa) \in C(a, b],\left(\frac{\varkappa^{\rho}-a^{\rho}}{\rho}\right)^{\gamma} g(\varkappa) \in C[a, b]\right\}, \\
& C_{\rho, \gamma}^{n}[a, b] \\
& \quad=\left\{\left(\varkappa^{1-\rho} \frac{d}{d \varkappa}\right)^{n} g(\varkappa) \in C_{\rho, \gamma}[a, b],\left(\varkappa^{1-\rho} \frac{d}{d \varkappa}\right)^{k} g(\varkappa) \in C[a, b], k=0, \ldots, n-1\right\},
\end{aligned}
$$

with the norms

$$
\|g\|_{C_{\rho, \gamma}}=\left\|\left(\frac{\varkappa^{\rho}-a^{\rho}}{\rho}\right)^{\gamma} g(\varkappa)\right\|_{C}
$$


and

$$
\|g\|_{C_{\rho, \gamma}^{n}}=\left\|\left(\varkappa^{1-\rho} \frac{d}{d \varkappa}\right)^{n} g\right\|_{C_{\rho, \gamma}}+\sum_{j=0}^{n-1}\left\|\left(\varkappa^{1-\rho} \frac{d}{d \varkappa}\right)^{j} g\right\|_{C},
$$

respectively.

Here we present some requisite definitions, notation and properties of the generalized fractional integral and derivative.

Definition 2.2 ([5]) The generalized fractional integral and derivative are defined, respectively, by

$$
\rho \mathfrak{I}_{a}^{\vartheta_{1}} g(\varkappa)=\frac{\rho^{1-\vartheta_{1}}}{\Gamma\left(\vartheta_{1}\right)} \int_{a}^{\varkappa}\left(\varkappa^{\rho}-t^{\rho}\right)^{\vartheta_{1}-1} t^{\rho-1} g(t) d t, \quad \vartheta_{1}>0, \rho>0,
$$

and

$$
\begin{aligned}
\rho \mathfrak{D}_{a}^{\vartheta_{1}} g(\varkappa) & =\delta_{\rho}^{n}\left({ }^{\rho} \mathfrak{I}_{a}^{n-\vartheta_{1}} g\right)(\varkappa) \\
& =\frac{\rho^{1+\vartheta_{1}-n}}{\Gamma\left(n-\vartheta_{1}\right)}\left(\varkappa^{1-\rho} \frac{d}{d \varkappa}\right)^{n} \int_{a}^{\varkappa}\left(\varkappa^{\rho}-t^{\rho}\right)^{n-\vartheta_{1}-1} t^{\rho-1} g(t) d t, \quad \vartheta_{1}>0, \rho>0,
\end{aligned}
$$

where

$$
n=-\left[-\vartheta_{1}\right], \quad \delta_{\rho}^{n}=\left(\varkappa^{1-\rho} \frac{d}{d \varkappa}\right)^{n}
$$

The generalized fractional integral and derivative (Definition 2.2) satisfy the following properties.

Property 2.3 ([33]) If $\vartheta_{1} \geq 0, \rho>0$ and $\vartheta_{2}>0$, then

$$
\begin{aligned}
& \rho \mathfrak{I}_{a}^{\vartheta_{1}}\left(\frac{\varkappa^{\rho}-a^{\rho}}{\rho}\right)^{\vartheta_{2}}=\frac{\Gamma\left(\vartheta_{2}+1\right)}{\Gamma\left(\vartheta_{2}+\vartheta_{1}+1\right)}\left(\frac{\varkappa^{\rho}-a^{\rho}}{\rho}\right)^{\vartheta_{2}+\vartheta_{1}}, \quad \varkappa>a, \\
& \rho \mathfrak{D}_{a}^{\vartheta_{1}}\left(\frac{\varkappa^{\rho}-a^{\rho}}{\rho}\right)^{\vartheta_{2}}=\frac{\Gamma\left(\vartheta_{2}+1\right)}{\Gamma\left(\vartheta_{2}-\vartheta_{1}+1\right)}\left(\frac{\varkappa^{\rho}-a^{\rho}}{\rho}\right)^{\vartheta_{2}-\vartheta_{1}}, \quad \varkappa>a .
\end{aligned}
$$

Property $2.4([33])$ Let $\vartheta_{1}, \rho, \vartheta_{2}>0$ and $0 \leq \mu<1$. If $g \in C_{\mu, \rho}[a, b]$, then

$$
\rho \mathfrak{I}_{a}^{\vartheta_{1} \rho} \mathfrak{I}_{a}^{\vartheta_{2}} g(\varkappa)={ }^{\rho} \mathfrak{I}_{a}^{\vartheta_{1}+\vartheta_{2}} g(\varkappa), \quad \varkappa>a .
$$

Property $2.5([5])$ Let $\vartheta_{1}>\vartheta_{2}>0$. If $g \in C_{\mu, \rho}[a, b]$, then

$$
\rho \mathfrak{D}_{a}^{\vartheta_{2} \rho} \mathfrak{I}_{a}^{\vartheta_{1}} g(\varkappa)={ }^{\rho} \mathfrak{I}_{a}^{\vartheta_{1}-\vartheta_{2}} g(\varkappa), \quad \varkappa>a
$$

Property 2.6 ([5]) If $g \in C_{\mu, \rho}[a, b]$ and $\vartheta_{1}>0$, then

$$
\rho \mathfrak{D}_{a}^{\vartheta_{1} \rho} \mathfrak{I}_{a}^{\vartheta_{1}} g=g, \quad \varkappa \in(a, b] .
$$


Theorem 2.7 ([12]) Let $0<\vartheta_{1}<1, \rho>0$ and $0 \leq \gamma<1$. If $g \in C_{\rho, \gamma}[a, b]$ and ${ }^{\rho} \mathfrak{I}_{a}^{1-\vartheta_{1}} g \in$ $C_{\rho, \gamma}^{1}[a, b]$, then

$$
\rho \mathfrak{I}_{a}^{\vartheta_{1} \rho} \mathfrak{D}_{a}^{\vartheta_{1}} g(\varkappa)=g(\varkappa)-\frac{\left(\rho \mathfrak{I}_{a}^{1-\vartheta_{1}} g\right)(a)}{\Gamma\left(\vartheta_{1}\right)}\left(\frac{\varkappa^{\rho}-a^{\rho}}{\rho}\right)^{\vartheta_{1}-1} .
$$

Next, we prove the following useful lemma.

Lemma 2.8 If $g \in C_{1-\vartheta_{1}, \rho}[a, b],{ }^{\rho} \mathfrak{I}_{a}^{1-\vartheta_{1}} g \in C_{1-\vartheta_{1}, \rho}^{1}[a, b], 0<\vartheta_{1}<1$, then, for $0 \leq \vartheta_{2}<\vartheta_{1}<1$, we have

$$
\rho \mathfrak{D}_{a}^{\vartheta_{2}} g(\varkappa)={ }^{\rho} \mathfrak{I}_{a}^{\vartheta_{1}-\vartheta_{2}} \rho \mathfrak{D}_{a}^{\vartheta_{1}} g(\varkappa)+\frac{\rho \mathfrak{I}_{a}^{1-\vartheta_{1}} g(a)}{\Gamma\left(\vartheta_{1}-\vartheta_{2}\right)}\left(\frac{\varkappa^{\rho}-a^{\rho}}{\rho}\right)^{\vartheta_{1}-\vartheta_{2}-1}, \quad \varkappa \in(a, b] .
$$

Proof By Theorem 2.7, we have

$$
\rho \mathfrak{I}_{a}^{\vartheta_{1} \rho} \mathfrak{D}_{a}^{\vartheta_{1}} g(\varkappa)=g(\varkappa)-\frac{\left(\rho \mathfrak{I}_{a}^{1-\vartheta_{1}} g\right)(a)}{\Gamma\left(\vartheta_{1}\right)}\left(\frac{\varkappa^{\rho}-a^{\rho}}{\rho}\right)^{\vartheta_{1}-1}, \quad \varkappa>a .
$$

Applying ${ }^{\rho} \mathfrak{D}_{a}^{\vartheta_{2}}$ to (2.1), using Properties 2.3 and 2.5, we obtain

$$
\rho \mathfrak{D}_{a}^{\vartheta_{2}} g(\varkappa)={ }^{\rho} \mathfrak{I}_{a}^{\vartheta_{1}-\vartheta_{2} \rho} \mathfrak{D}_{a}^{\vartheta_{1}} g(\varkappa)+\frac{\rho \mathfrak{I}_{a}^{1-\vartheta_{1}} g(a)}{\Gamma\left(\vartheta_{1}-\vartheta_{2}\right)}\left(\frac{\varkappa^{\rho}-a^{\rho}}{\rho}\right)^{\vartheta_{1}-\vartheta_{2}-1}, \quad \varkappa \in(a, b] .
$$

We recall some basic well-known results.

Theorem 2.9 (Bihari inequality, [34]) Let $v$ and $g$ be continuous nonnegative functions defined on $[0, \infty)$. Let $z(v)$ be a continuous nondecreasing function defined on $[0, \infty)$ and $z(v)>0$ on $(0, \infty)$. If

$$
v(\varkappa) \leq c+\int_{0}^{\varkappa} g(s) z(v(s)) d s
$$

for $\varkappa \in[0, \infty)$, where $c \geq 0$, then

$$
v(\varkappa) \leq \mathfrak{F}^{-1}\left(\mathfrak{F}(c)+\int_{0}^{\varkappa} g(s) d s\right)
$$

where $\mathfrak{F}^{-1}$ is the inverse function of

$$
\mathfrak{F}(\varkappa)=\int_{\varkappa_{0}}^{\varkappa} \frac{d s}{z(s)}, \quad \varkappa>0, \varkappa_{0}>0 .
$$

Lemma 2.10 ([35, 36]) For a nonnegativea and $b$, we have

$$
a^{r}+b^{r} \leq(a+b)^{r} \leq 2^{r-1}\left(a^{r}+b^{r}\right), \quad r \geq 1
$$

and

$$
2^{r-1}\left(a^{r}+b^{r}\right) \leq(a+b)^{r} \leq a^{r}+b^{r}, \quad 1 \geq r \geq 0 .
$$


Kassim et al. Advances in Difference Equations

(2021) $2021: 376$

Page 6 of 20

3 Stability

We discuss the problem (1.1) with the following suppositions.

(Al) $\mathfrak{F}(\varkappa, v, w):(a, \infty) \times \mathbb{R}^{2} \rightarrow \mathbb{R}$ is a function such that $\mathfrak{F}(., v(\cdot), w(\cdot)) \in C_{1-\vartheta_{1}, \rho}[a, \infty)$ for every $v, w \in C_{1-\vartheta_{1}, \rho}[a, \infty)$.

(A2) There is continuous functions $\varphi_{k}, k=1,2, h:[a, \infty) \rightarrow[0, \infty)$, such that

$$
\begin{aligned}
|\mathfrak{F}(\varkappa, v, w)| \leq & \left(\frac{\varkappa^{\rho}-a^{\rho}}{\rho}\right)^{\gamma} e^{-\delta\left(\varkappa^{\rho}-a^{\rho}\right)} h(\varkappa) \\
& \times \varphi_{1}\left(\left(\frac{\varkappa^{\rho}-a^{\rho}}{\rho}\right)^{1-\vartheta_{1}}|v|\right) \varphi_{2}\left(\left(\frac{\varkappa^{\rho}-a^{\rho}}{\rho}\right)^{1-\left(\vartheta_{1}-\vartheta_{2}\right)}|w|\right),
\end{aligned}
$$

where $\varphi_{k}, k=1,2$, are nondecreasing functions and $\gamma \in \mathbb{R}$.

(A3) There are two continuous functions $\varphi_{k}, h_{k}:[a, \infty) \rightarrow[0, \infty), k=1,2$, such that

$$
\begin{aligned}
|\mathfrak{F}(\varkappa, v, w)| \leq & \left(\frac{\varkappa^{\rho}-a^{\rho}}{\rho}\right)^{\gamma_{1}} e^{-\delta_{1}\left(\varkappa^{\rho}-a^{\rho}\right)} h_{1}(\varkappa) \varphi_{1}\left(\left(\frac{\varkappa^{\rho}-a^{\rho}}{\rho}\right)^{1-\vartheta_{1}}|v|\right) \\
& +\left(\frac{\varkappa^{\rho}-a^{\rho}}{\rho}\right)^{\gamma_{2}} e^{-\delta_{2}\left(\varkappa^{\rho}-a^{\rho}\right)} h_{2}(\varkappa) \\
& \times \varphi_{2}\left(\left(\frac{\varkappa^{\rho}-a^{\rho}}{\rho}\right)^{1-\left(\vartheta_{1}-\vartheta_{2}\right)}|w|\right),
\end{aligned}
$$

where $\varphi_{k}, k=1,2$, are nondecreasing functions and $\gamma_{k} \in \mathbb{R}, k=1,2$. The following general interesting inequality is proved.

Lemma 3.1 For $\lambda, v, \rho>0, \omega>0$, we have

$$
\left(\varkappa^{\rho}-a^{\rho}\right)^{1-\nu} \int_{a}^{\varkappa}\left(\varkappa^{\rho}-s^{\rho}\right)^{\nu-1}\left(s^{\rho}-a^{\rho}\right)^{\lambda-1} e^{-\omega\left(s^{\rho}-a^{\rho}\right)} s^{\rho-1} d s \leq C, \quad \varkappa>a,
$$

where

$$
C=\rho^{-1} \max \left(1,2^{1-v}\right) \omega^{-\lambda} \Gamma(\lambda)\left(1+\frac{\lambda(\lambda+1)}{v}\right) .
$$

Proof Put

$$
L(\varkappa):=\left(\varkappa^{\rho}-a^{\rho}\right)^{1-\nu} \int_{a}^{\varkappa}\left(\varkappa^{\rho}-s^{\rho}\right)^{\nu-1}\left(s^{\rho}-a^{\rho}\right)^{\lambda-1} e^{-\omega\left(s^{\rho}-a^{\rho}\right)} s^{\rho-1} d s, \quad \varkappa>a .
$$

Let $r=\frac{s^{\rho}-a^{\rho}}{\varkappa^{\rho}-a^{\rho}}$. Then $s^{\rho}=r\left(\varkappa^{\rho}-a^{\rho}\right)+a^{\rho}$ and $s^{\rho-1} d s=\frac{1}{\rho}\left(\varkappa^{\rho}-a^{\rho}\right) d r$. Therefore

$$
\begin{aligned}
L(\varkappa)= & \left(\varkappa^{\rho}-a^{\rho}\right)^{1-\nu} \int_{0}^{1}\left(\varkappa^{\rho}-a^{\rho}-\left(\varkappa^{\rho}-a^{\rho}\right) r\right)^{\nu-1}\left(\left(\varkappa^{\rho}-a^{\rho}\right) r\right)^{\lambda-1} \\
& \times e^{-\omega r\left(\varkappa^{\rho}-a^{\rho}\right)} \frac{1}{\rho}\left(\varkappa^{\rho}-a^{\rho}\right) d r \\
= & \frac{1}{\rho}\left(\varkappa^{\rho}-a^{\rho}\right)^{\lambda} \int_{0}^{1}(1-r)^{\nu-1} r^{\lambda-1} e^{-\omega r\left(\varkappa^{\rho}-a^{\rho}\right)} d r \\
= & \frac{1}{\rho}\left(\varkappa^{\rho}-a^{\rho}\right)^{\lambda} \int_{0}^{1 / 2}(1-r)^{\nu-1} r^{\lambda-1} e^{-\omega r\left(\varkappa^{\rho}-a^{\rho}\right)} d r
\end{aligned}
$$


Kassim et al. Advances in Difference Equations

(2021) 2021:376

Page 7 of 20

$$
\begin{aligned}
& +\frac{1}{\rho}\left(\varkappa^{\rho}-a^{\rho}\right)^{\lambda} \int_{1 / 2}^{1}(1-r)^{\nu-1} r^{\lambda-1} e^{-\omega r\left(\varkappa^{\rho}-a^{\rho}\right)} d r \\
\leq & \rho^{-1} \max \left(1,2^{1-\nu}\right)\left(\varkappa^{\rho}-a^{\rho}\right)^{\lambda} \int_{0}^{1 / 2} r^{\lambda-1} e^{-\omega r\left(\varkappa^{\rho}-a^{\rho}\right)} d r \\
& +\rho^{-1}\left(\varkappa^{\rho}-a^{\rho}\right)^{\lambda} \int_{1 / 2}^{1}(1-r)^{\nu-1} r^{\lambda-1} e^{-\omega r\left(\varkappa^{\rho}-a^{\rho}\right)} d r .
\end{aligned}
$$

Let $u=\omega r\left(\varkappa^{\rho}-a^{\rho}\right)$, then $d u=\omega\left(\varkappa^{\rho}-a^{\rho}\right) d r$ and

$$
\left(\varkappa^{\rho}-a^{\rho}\right)^{\lambda} \int_{0}^{1 / 2} r^{\lambda-1} e^{-\omega r\left(\varkappa^{\rho}-a^{\rho}\right)} d r \leq \omega^{-\lambda} \int_{0}^{\infty} u^{\lambda-1} e^{-u} d u=\omega^{-\lambda} \Gamma(\lambda) .
$$

If $\omega r\left(\varkappa^{\rho}-a^{\rho}\right) \geq 1$, then

$$
e^{\omega r\left(\varkappa^{\rho}-a^{\rho}\right)} \geq \frac{\left(\omega r\left(\varkappa^{\rho}-a^{\rho}\right)\right)^{[\lambda]+1}}{([\lambda]+1) !}=\frac{\left(\omega r\left(\varkappa^{\rho}-a^{\rho}\right)\right)^{[\lambda]+1}}{\Gamma([\lambda]+2)} \geq \frac{\left(\omega r\left(\varkappa^{\rho}-a^{\rho}\right)\right)^{\lambda}}{\Gamma(\lambda+2)} .
$$

Therefore, when $1 / 2<r \leq 1$

$$
r^{\lambda-1} e^{-\omega r\left(\varkappa^{\rho}-a^{\rho}\right)} \leq \frac{r^{\lambda-1} \Gamma(\lambda+2)}{\left(\omega r\left(\varkappa^{\rho}-a^{\rho}\right)\right)^{\lambda}} \leq \frac{2 \omega^{-\lambda} \Gamma(\lambda+2)}{\left(\varkappa^{\rho}-a^{\rho}\right)^{\lambda}}
$$

and consequently

$$
\begin{aligned}
& \rho^{-1}\left(\varkappa^{\rho}-a^{\rho}\right)^{\lambda} \int_{1 / 2}^{1}(1-r)^{\nu-1} r^{\lambda-1} e^{-\omega r\left(\varkappa^{\rho}-a^{\rho}\right)} d r \\
& \leq 2 \omega^{-\lambda} \rho^{-1} \Gamma(\lambda+2) \int_{1 / 2}^{1}(1-r)^{\nu-1} d r=\frac{2^{1-\nu} \omega^{-\lambda} \Gamma(\lambda+2)}{\rho \nu} .
\end{aligned}
$$

When $\omega r\left(\varkappa^{\rho}-a^{\rho}\right)<1$, we have $e^{\omega r\left(\varkappa^{\rho}-a^{\rho}\right)} \geq 1>\left(\omega r\left(\varkappa^{\rho}-a^{\rho}\right)\right)^{\lambda}$ and thus

$$
\begin{aligned}
& \rho^{-1}\left(\varkappa^{\rho}-a^{\rho}\right)^{\lambda} \int_{1 / 2}^{1}(1-r)^{\nu-1} r^{\lambda-1} e^{-\omega r\left(\varkappa^{\rho}-a^{\rho}\right)} d r \\
& <\rho^{-1}\left(\varkappa^{\rho}-a^{\rho}\right)^{\lambda} \int_{1 / 2}^{1}(1-r)^{\nu-1} r^{\lambda-1}\left(\omega r\left(\varkappa^{\rho}-a^{\rho}\right)\right)^{-\lambda} d r \\
& <2 \rho^{-1} \omega^{-\lambda} \int_{1 / 2}^{1}(1-r)^{\nu-1} d r=2^{1-\nu} \frac{\omega^{-\lambda}}{\rho \nu} .
\end{aligned}
$$

Take into consideration (3.3)-(3.6) we conclude that

$$
\begin{aligned}
L(\varkappa) & \leq \rho^{-1} \max \left(1,2^{1-\nu}\right) \omega^{-\lambda} \Gamma(\lambda)+\frac{2^{1-v} \omega^{-\lambda} \Gamma(\lambda+2)}{\rho v} \\
& \leq \rho^{-1} \max \left(1,2^{1-v}\right) \omega^{-\lambda} \Gamma(\lambda)\left(1+\frac{\lambda(\lambda+1)}{v}\right) .
\end{aligned}
$$

We prove a useful inequality enjoyed by solutions of Problem (1.1).

Lemma 3.2 Suppose that $\mathfrak{F}$ satisfies (A1), (A2) and $\varpi(\varkappa)$ is solution of (1.1). Then

$$
\max \left\{\left(\frac{\varkappa^{\rho}-a^{\rho}}{\rho}\right)^{1-\vartheta_{1}}|\varpi(\varkappa)|,\left(\frac{\varkappa^{\rho}-a^{\rho}}{\rho}\right)^{1-\left(\vartheta_{1}-\vartheta_{2}\right)}\left|{ }^{\rho} \mathfrak{D}_{a}^{\vartheta_{2}} \varpi(\varkappa)\right|\right\}
$$


Kassim et al. Advances in Difference Equations

(2021) 2021:376

Page 8 of 20

$$
\leq z(\varkappa), \quad \varkappa>a,
$$

where

$$
\begin{aligned}
z(\varkappa)= & K_{1}+K_{2}\left\{\int_{a}^{\varkappa} h^{q}(s) \varphi_{1}^{q}\left(\left(\frac{s^{\rho}-a^{\rho}}{\rho}\right)^{1-\vartheta_{1}}|\varpi(s)|\right) \varphi_{2}^{q}\right. \\
& \left.\times \varphi_{2}^{q}\left(\left(\frac{s^{\rho}-a^{\rho}}{\rho}\right)^{1-\left(\vartheta_{1}-\vartheta_{2}\right)}\left|{ }^{\rho} \mathfrak{D}_{a}^{\vartheta_{2}} \varpi(s)\right|\right) \frac{d s}{s^{1-\rho}}\right\}^{1 / q}
\end{aligned}
$$

when $h \in L_{q}(a, \infty)$ for some $q>\frac{1}{\vartheta_{1}-\vartheta_{2}}, \gamma>\frac{1}{q}-1$,

$$
K_{1}=|b| \max \left\{\frac{1}{\Gamma\left(\vartheta_{1}\right)}, \frac{1}{\Gamma\left(\vartheta_{1}-\vartheta_{2}\right)}\right\} \quad \text { and } \quad K_{2}=\rho^{-\gamma} \max \left\{\frac{C_{1}^{\prime}}{\Gamma\left(\vartheta_{1}\right)}, \frac{C_{2}^{\prime}}{\Gamma\left(\vartheta_{1}-\vartheta_{2}\right)}\right\} \text {, }
$$

where $p+q=p q$,

$$
C_{1}^{\prime}=\left[\frac{1}{\rho} \max \left\{1,2^{p\left(1-\vartheta_{1}\right)}\right\}(p \delta)^{-(p \gamma+1)} \Gamma(p \gamma+1)\left(1+\frac{(p \gamma+1)(p \gamma+2)}{p\left(1-\vartheta_{1}\right)+1}\right)\right]^{1 / p}
$$

and

$$
C_{2}^{\prime}=\left[\frac{1}{\rho} \max \left\{1,2^{p\left(1-\vartheta_{1}+\vartheta_{2}\right)}\right\}\left(1+\frac{(p \gamma+1)(p \gamma+2)}{p\left(\vartheta_{1}-\vartheta_{2}-1\right)+1}\right)(p \delta)^{-(p \gamma+1)} \Gamma(p \gamma+1)\right]^{1 / p} .
$$

Proof Applying ${ }^{\rho} \mathfrak{I}_{a}^{\vartheta_{1}}$ to (1.1) and using Theorem 2.7, we find that

$$
\begin{aligned}
\varpi(\varkappa)= & \frac{b}{\Gamma\left(\vartheta_{1}\right)}\left(\frac{\varkappa^{\rho}-a^{\rho}}{\rho}\right)^{\vartheta_{1}-1} \\
& +\frac{1}{\Gamma\left(\vartheta_{1}\right)} \int_{a}^{\varkappa}\left(\frac{\varkappa^{\rho}-s^{\rho}}{\rho}\right)^{\vartheta_{1}-1} \mathfrak{F}\left(s, \varpi(s),{ }^{\rho} \mathfrak{D}_{a}^{\vartheta_{2}} \varpi(s)\right) \frac{d s}{s^{1-\rho}}
\end{aligned}
$$

and using (3.1), we obtain

$$
\begin{aligned}
& \left(\frac{\varkappa^{\rho}-a^{\rho}}{\rho}\right)^{1-\vartheta_{1}}|\varpi(\varkappa)| \\
& \leq K_{1}+\frac{1}{\Gamma\left(\vartheta_{1}\right)}\left(\frac{\varkappa^{\rho}-a^{\rho}}{\rho}\right)^{1-\vartheta_{1}} \\
& \quad \times \int_{a}^{\varkappa}\left\{\left(\frac{\varkappa^{\rho}-s^{\rho}}{\rho}\right)^{\vartheta_{1}-1}\left(\frac{s^{\rho}-a^{\rho}}{\rho}\right)^{\gamma} e^{-\delta\left(s^{\rho}-a^{\rho}\right)} h(s)\right. \\
& \quad \times \varphi_{1}\left[\left(\frac{s^{\rho}-a^{\rho}}{\rho}\right)^{1-\vartheta_{1}}|\varpi(s)|\right] \\
& \left.\quad \times \varphi_{2}\left[\left(\frac{s^{\rho}-a^{\rho}}{\rho}\right)^{1-\left(\vartheta_{1}-\vartheta_{2}\right)}\left|\rho^{\vartheta_{a}} \varpi(s)\right|\right] \frac{d s}{s^{1-\rho}}\right\}, \quad \varkappa>a .
\end{aligned}
$$

Now, the Hölder inequality yields

$$
J_{1}:=\int_{a}^{\varkappa}\left\{\left(\frac{\varkappa^{\rho}-s^{\rho}}{\rho}\right)^{\vartheta_{1}-1}\left(\frac{s^{\rho}-a^{\rho}}{\rho}\right)^{\gamma} e^{-\delta\left(s^{\rho}-a^{\rho}\right)}\right.
$$


Kassim et al. Advances in Difference Equations

(2021) 2021:376

Page 9 of 20

$$
\begin{aligned}
& \left.\times h(s) \varphi_{1}\left[\left(\frac{s^{\rho}-a^{\rho}}{\rho}\right)^{1-\vartheta_{1}}|\varpi(s)|\right] \varphi_{2}\left[\left(\frac{s^{\rho}-a^{\rho}}{\rho}\right)^{1-\left(\vartheta_{1}-\vartheta_{2}\right)}\left|{ }^{\rho} \mathfrak{D}_{a}^{\vartheta_{2}} \varpi(s)\right|\right] \frac{d s}{s^{1-\rho}}\right\} \\
& \leq\left[\int_{a}^{\varkappa}\left(\frac{\varkappa^{\rho}-s^{\rho}}{\rho}\right)^{p\left(\vartheta_{1}-1\right)}\left(\frac{s^{\rho}-a^{\rho}}{\rho}\right)^{p \gamma} e^{-p \delta\left(s^{\rho}-a^{\rho}\right)} \frac{d s}{s^{1-\rho}}\right]^{\frac{1}{p}} \\
& \times\left\{\int_{a}^{\varkappa} h^{q}(s) \varphi_{1}^{q}\left[\left(\frac{s^{\rho}-a^{\rho}}{\rho}\right)^{1-\vartheta_{1}}|\varpi(s)|\right]\right. \\
& \left.\times \varphi_{2}^{q}\left[\left(\frac{s^{\rho}-a^{\rho}}{\rho}\right)^{1-\left(\vartheta_{1}-\vartheta_{2}\right)}\left|\rho \mathfrak{D}_{a}^{\vartheta_{2}} \varpi(s)\right|\right] \frac{d s}{s^{1-\rho}}\right\}^{\frac{1}{q}} \\
& \leq \rho^{1-\vartheta_{1}-\gamma}\left[\int_{a}^{\varkappa}\left(\varkappa^{\rho}-s^{\rho}\right)^{p\left(\vartheta_{1}-1\right)}\left(s^{\rho}-a^{\rho}\right)^{p \gamma} e^{-p \delta\left(s^{\rho}-a^{\rho}\right)} \frac{d s}{s^{1-\rho}}\right]^{\frac{1}{p}} \\
& \times\left\{\int_{a}^{\varkappa} h^{q}(s) \varphi_{1}^{q}\left[\left(\frac{s^{\rho}-a^{\rho}}{\rho}\right)^{1-\vartheta_{1}}|\varpi(s)|\right]\right. \\
& \left.\times \varphi_{2}^{q}\left[\left(\frac{s^{\rho}-a^{\rho}}{\rho}\right)^{1-\left(\vartheta_{1}-\vartheta_{2}\right)}\left|\mathfrak{D}_{a}^{\vartheta_{2}} \varpi(s)\right|\right] \frac{d s}{s^{1-\rho}}\right\}^{\frac{1}{q}}, \quad \varkappa>a \text {. }
\end{aligned}
$$

Now, as $q>\frac{1}{\vartheta_{1}-\vartheta_{2}}$ implies $p\left(\vartheta_{1}-1\right)>-1$ and $p \gamma>-1$, we apply Lemma 3.1 to get

$$
\begin{aligned}
J_{1} \leq & \rho^{1-\vartheta_{1}-\gamma} C_{1}^{\prime}\left(\varkappa^{\rho}-a^{\rho}\right)^{\vartheta_{1}-1} \\
& \times\left\{\int_{a}^{\varkappa} h^{q}(s) \varphi_{1}^{q}\left[\left(\frac{s^{\rho}-a^{\rho}}{\rho}\right)^{1-\vartheta_{1}}|\varpi(s)|\right]\right. \\
& \left.\times \varphi_{2}^{q}\left[\left(\frac{s^{\rho}-a^{\rho}}{\rho}\right)^{1-\left(\vartheta_{1}-\vartheta_{2}\right)}\left|\mathfrak{D}_{a}^{\vartheta_{2}} \varpi(s)\right|\right] \frac{d s}{s^{1-\rho}}\right\}^{\frac{1}{q}}, \quad \varkappa>a .
\end{aligned}
$$

Combining (3.10) and (3.11) we conclude that

$$
\begin{aligned}
\left(\frac{\varkappa^{\rho}-a^{\rho}}{\rho}\right)^{1-\vartheta_{1}}|\varpi(\varkappa)| \leq & K_{1}+K_{2}\left\{\int_{a}^{\varkappa} h^{q}(s) \varphi_{1}^{q}\left[\left(\frac{s^{\rho}-a^{\rho}}{\rho}\right)^{1-\vartheta_{1}}|\varpi(s)|\right]\right. \\
& \left.\times \varphi_{2}^{q}\left[\left(\frac{s^{\rho}-a^{\rho}}{\rho}\right)^{1-\left(\vartheta_{1}-\vartheta_{2}\right)}\left|\mathfrak{D}_{a}^{\vartheta_{2}} \varpi(s)\right|\right] \frac{d s}{s^{1-\rho}}\right\}^{\frac{1}{q}},
\end{aligned}
$$

for $\varkappa>a$. By using Lemma 2.8, we have

$$
\begin{aligned}
& \rho \mathfrak{D}_{a}^{\vartheta_{2}} \varpi(\varkappa) \\
&={ }^{\rho} \mathfrak{I}_{a}^{\vartheta_{1}-\vartheta_{2} \rho} \mathfrak{D}_{a}^{\vartheta_{1}} \varpi(\varkappa)+\frac{b}{\Gamma\left(\vartheta_{1}-\vartheta_{2}\right)}\left(\frac{\varkappa^{\rho}-a^{\rho}}{\rho}\right)^{\vartheta_{1}-\vartheta_{2}-1} \\
&=\frac{b}{\Gamma\left(\vartheta_{1}-\vartheta_{2}\right)}\left(\frac{\varkappa^{\rho}-a^{\rho}}{\rho}\right)^{\vartheta_{1}-\vartheta_{2}-1} \\
&+\frac{1}{\Gamma\left(\vartheta_{1}-\vartheta_{2}\right)} \int_{a}^{\varkappa}\left(\frac{\varkappa^{\rho}-s^{\rho}}{\rho}\right)^{\vartheta_{1}-\vartheta_{2}-1} \rho^{\rho} \mathfrak{D}_{a}^{\vartheta_{1}} \varpi(s) \frac{d s}{s^{1-\rho}} \\
&=\frac{b}{\Gamma\left(\vartheta_{1}-\vartheta_{2}\right)}\left(\frac{\varkappa^{\rho}-a^{\rho}}{\rho}\right)^{\vartheta_{1}-\vartheta_{2}-1} \\
&+\frac{1}{\Gamma\left(\vartheta_{1}-\vartheta_{2}\right)} \int_{a}^{\varkappa}\left(\frac{\varkappa^{\rho}-s^{\rho}}{\rho}\right)^{\vartheta_{1}-\vartheta_{2}-1} \mathfrak{F}\left(s, \varpi(s),{ }^{\rho} \mathfrak{D}_{a}^{\vartheta_{2}} \varpi(s)\right) \frac{d s}{s^{1-\rho}}, \quad \varkappa>a
\end{aligned}
$$


Kassim et al. Advances in Difference Equations

(2021) 2021:376

Page 10 of 20

and in view of (3.1)

$$
\begin{aligned}
& \left(\frac{\varkappa^{\rho}-a^{\rho}}{\rho}\right)^{1-\left(\vartheta_{1}-\vartheta_{2}\right)}||^{\rho} \mathfrak{D}_{a}^{\vartheta_{2}} \varpi(\varkappa) \mid \\
& \leq K_{1}+\frac{1}{\Gamma\left(\vartheta_{1}-\vartheta_{2}\right)}\left(\frac{\varkappa^{\rho}-a^{\rho}}{\rho}\right)^{1-\left(\vartheta_{1}-\vartheta_{2}\right)} \int_{a}^{\varkappa}\left\{\left(\frac{\varkappa^{\rho}-s^{\rho}}{\rho}\right)^{\vartheta_{1}-\vartheta_{2}-1}\left(\frac{s^{\rho}-a^{\rho}}{\rho}\right)^{\gamma}\right. \\
& \quad \times e^{-\delta\left(s^{\rho}-a^{\rho}\right)} h(s) \varphi_{1}\left[\left(\frac{s^{\rho}-a^{\rho}}{\rho}\right)^{1-\vartheta_{1}}|\varpi(s)|\right] \\
& \left.\quad \times \varphi_{2}\left[\left(\frac{s^{\rho}-a^{\rho}}{\rho}\right)^{1-\left(\vartheta_{1}-\vartheta_{2}\right)}\left|\rho \mathfrak{D}_{a}^{\vartheta_{2}} \varpi(s)\right|\right] \frac{d s}{s^{1-\rho}}\right\}
\end{aligned}
$$

for $\varkappa>a$. By using the Hölder inequality, we see that

$$
\begin{aligned}
J_{2}:= & \int_{a}^{\varkappa}\left\{\left(\frac{\varkappa^{\rho}-s^{\rho}}{\rho}\right)^{\vartheta_{1}-\vartheta_{2}-1}\left(\frac{s^{\rho}-a^{\rho}}{\rho}\right)^{\gamma}\right. \\
& \times e^{-\delta\left(s^{\rho}-a^{\rho}\right)} h(s) \varphi_{1}\left[\left(\frac{s^{\rho}-a^{\rho}}{\rho}\right)^{1-\vartheta_{1}}|\varpi(s)|\right] \\
& \left.\times \varphi_{2}\left[\left(\frac{s^{\rho}-a^{\rho}}{\rho}\right)^{1-\left(\vartheta_{1}-\vartheta_{2}\right)}\left|{ }^{\rho} \mathfrak{D}_{a}^{\vartheta_{2}} \varpi(s)\right|\right] \frac{d s}{s^{1-\rho}}\right\} \\
\leq & {\left[\int_{a}^{\varkappa}\left(\frac{\varkappa^{\rho}-s^{\rho}}{\rho}\right)^{p\left(\vartheta_{1}-\vartheta_{2}-1\right)}\left(\frac{s^{\rho}-a^{\rho}}{\rho}\right)^{p \gamma} e^{-p \delta\left(s^{\rho}-a^{\rho}\right)} \frac{d s}{s^{1-\rho}}\right]^{\frac{1}{p}} } \\
& \times\left\{\int_{a}^{\varkappa} h^{q}(s) \varphi_{1}^{q}\left[\left(\frac{s^{\rho}-a^{\rho}}{\rho}\right)^{1-\vartheta_{1}}|\varpi(s)|\right]\right. \\
& \left.\times \varphi_{2}^{q}\left[\left(\frac{s^{\rho}-a^{\rho}}{\rho}\right)^{1-\left(\vartheta_{1}-\vartheta_{2}\right)}\left|{ }^{\rho} \mathfrak{D}_{a}^{\vartheta_{2}} \varpi(s)\right|\right] \frac{d s}{s^{1-\rho}}\right\}^{\frac{1}{q}} \\
\leq & \rho^{1-\left(\vartheta_{1}-\vartheta_{2}\right)-\gamma}\left[\int_{a}^{\varkappa}\left(\varkappa^{\rho}-s^{\rho}\right)^{p\left(\vartheta_{1}-\vartheta_{2}-1\right)}\left(s^{\rho}-a^{\rho}\right)^{p \gamma} e^{-p \delta\left(s^{\rho}-a^{\rho}\right)} \frac{d s}{s^{1-\rho}}\right]^{\frac{1}{p}} \\
& \times\left\{\int_{a}^{\varkappa} h^{q}(s) \varphi_{1}^{q}\left[\left(\frac{s^{\rho}-a^{\rho}}{\rho}\right)^{1-\vartheta_{1}}|\varpi(s)|\right]\right. \\
& \left.\times \varphi_{2}^{q}\left[\left(\frac{s^{\rho}-a^{\rho}}{\rho}\right)^{1-\left(\vartheta_{1}-\vartheta_{2}\right)}||^{\rho} \mathfrak{D}_{a}^{\vartheta_{2}} \varpi(s) \mid\right] \frac{d s}{s^{1-\rho}}\right\}^{\frac{1}{q}},
\end{aligned}
$$

for $\varkappa>a$. Again by Lemma 3.1 (with $p\left(\vartheta_{1}-\vartheta_{2}-1\right)>-1$ and $p \gamma>-1$ ), we obtain

$$
\begin{aligned}
J_{2} \leq & \rho^{-\gamma} C_{2}^{\prime}\left(\frac{s^{\rho}-a^{\rho}}{\rho}\right)^{\vartheta_{1}-\vartheta_{2}-1}\left\{\int_{a}^{\varkappa} h^{q}(s) \varphi_{1}^{q}\left[\left(\frac{s^{\rho}-a^{\rho}}{\rho}\right)^{1-\vartheta_{1}}|\varpi(s)|\right]\right. \\
& \left.\times \varphi_{2}^{q}\left[\left(\frac{s^{\rho}-a^{\rho}}{\rho}\right)^{1-\left(\vartheta_{1}-\vartheta_{2}\right)}\left|{ }^{\rho} \mathfrak{D}_{a}^{\vartheta_{2}} \varpi(s)\right|\right] \frac{d s}{s^{1-\rho}}\right\}^{\frac{1}{q}},
\end{aligned}
$$

Combining (3.14) and (3.15), we arrive at

$$
\left.\left(\frac{\varkappa^{\rho}-a^{\rho}}{\rho}\right)^{1-\left(\vartheta_{1}-\vartheta_{2}\right)}\right|^{\rho} \mathfrak{D}_{a}^{\vartheta_{2}} \varpi(\varkappa) \mid
$$


Kassim et al. Advances in Difference Equations

(2021) 2021:376

Page 11 of 20

$$
\begin{aligned}
\leq & K_{1}+K_{2}\left\{\int_{a}^{\varkappa} h^{q}(s) \varphi_{1}^{q}\left[\left(\frac{s^{\rho}-a^{\rho}}{\rho}\right)^{1-\vartheta_{1}}|\varpi(s)|\right]\right. \\
& \left.\times \varphi_{2}^{q}\left[\left(\frac{s^{\rho}-a^{\rho}}{\rho}\right)^{1-\left(\vartheta_{1}-\vartheta_{2}\right)}\left|\rho \mathfrak{D}_{a}^{\vartheta_{2}} \varpi(s)\right|\right] \frac{d s}{s^{1-\rho}}\right\}^{\frac{1}{q}},
\end{aligned}
$$

for $\varkappa>a$. The relation (3.7) is an instant consequence of (3.12) and (3.16).

Lemma 3.3 Suppose that $\mathfrak{F}$ satisfies (A1), (A3) and $\varpi(\varkappa)$ is solution of (1.1). Then

$$
\begin{aligned}
& \max \left\{\left(\frac{\varkappa^{\rho}-a^{\rho}}{\rho}\right)^{1-\vartheta_{1}}|\varpi(\varkappa)|,\left(\frac{\varkappa^{\rho}-a^{\rho}}{\rho}\right)^{1-\left(\vartheta_{1}-\vartheta_{2}\right)}||^{\rho} \mathfrak{D}_{a}^{\vartheta_{2}} \varpi(\varkappa) \mid\right\} \\
& \leq z(\varkappa), \quad \varkappa>a,
\end{aligned}
$$

where

$$
\begin{aligned}
z(\varkappa)= & K_{1}+K_{2}\left\{\left[\int_{a}^{\varkappa} h_{1}^{q}(s) \varphi_{1}^{q}\left[\left(\frac{s^{\rho}-a^{\rho}}{\rho}\right)^{1-\vartheta_{1}}|\varpi(s)|\right] \frac{d s}{s^{1-\rho}}\right]^{1 / q}\right. \\
& \left.+\left[\int_{a}^{\varkappa} h_{2}^{q}(s) \varphi_{2}^{q}\left(\left(\frac{s^{\rho}-a^{\rho}}{\rho}\right)^{1-\left(\vartheta_{1}-\vartheta_{2}\right)}\left|\rho \mathfrak{D}_{a}^{\vartheta_{2}} \varpi(s)\right|\right) \frac{d s}{s^{1-\rho}}\right]^{1 / q}\right\}, \quad \varkappa>a,
\end{aligned}
$$

where $h_{k} \in L_{q}(a, \infty)$ for some $q>\frac{1}{\vartheta_{1}-\vartheta_{2}}, \gamma_{k}>\frac{1}{q}-1$, and $\delta_{k}>0, k=1,2$,

$$
\begin{aligned}
& K_{1}=|b| \max \left\{\frac{1}{\Gamma\left(\vartheta_{1}\right)}, \frac{1}{\Gamma\left(\vartheta_{1}-\vartheta_{2}\right)}\right\} \text { and } K_{2}=\max \left\{C_{3}, C_{3}^{\prime}\right\}, \\
& C_{3}=\frac{\rho^{-\gamma_{k}}}{\Gamma\left(\vartheta_{1}\right)} \max \left\{C_{1}, C_{2}\right\}, \\
& C_{k}=\left(\frac{1}{\rho} \max \left\{1,2^{p\left(1-\vartheta_{1}\right)}\right\} \Gamma\left(1+p \gamma_{k}\right)\left(\frac{\left(p \gamma_{k}+1\right)\left(p \gamma_{k}+2\right)}{p\left(\vartheta_{1}-1\right)+1}+1\right)\left(p \delta_{k}\right)^{-\left(1+p \gamma_{k}\right)}\right)^{\frac{1}{p}}, \\
& C_{3}^{\prime}=\frac{\rho^{-\gamma_{k}}}{\Gamma\left(\vartheta_{1}-\vartheta_{2}\right)} \max \left\{C_{1}^{\prime}, C_{2}^{\prime}\right\}, \\
& C_{k}^{\prime}=\left(\frac{1}{\rho} \max \left\{1,2^{p\left(1-\left(\vartheta_{1}-\vartheta_{2}\right)\right)}\right\} \Gamma\left(p \gamma_{k}+1\right)\left(1+\frac{\left(p \gamma_{k}+1\right)\left(p \gamma_{k}+2\right)}{p\left(\vartheta_{1}-\vartheta_{2}-1\right)+1}\right)\left(p \delta_{k}\right)^{-\left(p \gamma_{k}+1\right)}\right)^{\frac{1}{p}} .
\end{aligned}
$$

Proof Applying ${ }^{\rho} \mathfrak{I}_{a}^{\vartheta_{1}}$ to (1.1) and using Theorem 2.7, we find that

$$
\begin{aligned}
\varpi(\varkappa)= & \frac{b}{\Gamma\left(\vartheta_{1}\right)}\left(\frac{\varkappa^{\rho}-a^{\rho}}{\rho}\right)^{\vartheta_{1}-1}+\frac{1}{\Gamma\left(\vartheta_{1}\right)} \int_{a}^{\varkappa}\left(\frac{\varkappa^{\rho}-s^{\rho}}{\rho}\right)^{\vartheta_{1}-1} \\
& \times \mathfrak{F}\left(s, \varpi(s),{ }^{\rho} \mathfrak{D}_{a}^{\vartheta_{2}} \varpi(s)\right) \frac{d s}{s^{1-\rho}}
\end{aligned}
$$

for $\varkappa>a$. Multiplying (3.19) by $\left(\frac{\varkappa^{\rho}-a^{\rho}}{\rho}\right)^{1-\vartheta_{1}}$ and using (3.2), we get

$$
\begin{aligned}
& \left(\frac{\varkappa^{\rho}-a^{\rho}}{\rho}\right)^{1-\vartheta_{1}}|\varpi(\varkappa)| \\
& \leq K_{1}+\frac{1}{\Gamma\left(\vartheta_{1}\right)}\left(\frac{\varkappa^{\rho}-a^{\rho}}{\rho}\right)^{1-\vartheta_{1}}
\end{aligned}
$$


Kassim et al. Advances in Difference Equations

(2021) 2021:376

Page 12 of 20

$$
\begin{aligned}
& \times \int_{a}^{\varkappa}\left(\frac{\varkappa^{\rho}-s^{\rho}}{\rho}\right)^{\vartheta_{1}-1}\left(\frac{s^{\rho}-a^{\rho}}{\rho}\right)^{\gamma_{1}} e^{-\delta_{1}\left(s^{\rho}-a^{\rho}\right)} \\
& \times h_{1}(s) \varphi_{1}\left[\left(\frac{s^{\rho}-a^{\rho}}{\rho}\right)^{1-\vartheta_{1}}|\varpi(s)|\right] \frac{d s}{s^{1-\rho}} \\
& +\frac{1}{\Gamma\left(\vartheta_{1}\right)}\left(\frac{\varkappa^{\rho}-a^{\rho}}{\rho}\right)^{1-\vartheta_{1}} \int_{a}^{\varkappa}\left(\frac{\varkappa^{\rho}-s^{\rho}}{\rho}\right)^{\vartheta_{1}-1}\left(\frac{s^{\rho}-a^{\rho}}{\rho}\right)^{\gamma_{2}} e^{-\delta_{2}\left(s^{\rho}-a^{\rho}\right)} \\
& \times h_{2}(s) \varphi_{2}\left[\left(\frac{s^{\rho}-a^{\rho}}{\rho}\right)^{1-\left(\vartheta_{1}-\vartheta_{2}\right)}\left|{ }^{\rho} \mathfrak{D}_{a}^{\vartheta_{1}} \varpi(s)\right|\right] \frac{d s}{s^{1-\rho}} .
\end{aligned}
$$

From the Hölder inequality we have

$$
\begin{aligned}
\left(\frac{\varkappa^{\rho}-a^{\rho}}{\rho}\right)^{1-\vartheta_{1}}|\varpi(\varkappa)| \leq & K_{1}+\frac{1}{\Gamma\left(\vartheta_{1}\right)}\left(\frac{\varkappa^{\rho}-a^{\rho}}{\rho}\right)^{1-\vartheta_{1}} \\
& \times\left(\int_{a}^{\varkappa}\left(\frac{\varkappa^{\rho}-s^{\rho}}{\rho}\right)^{p\left(\vartheta_{1}-1\right)}\left(\frac{s^{\rho}-a^{\rho}}{\rho}\right)^{p \gamma_{1}} e^{-p \delta_{1}\left(s^{\rho}-a^{\rho}\right)} \frac{d s}{s^{1-\rho}}\right)^{\frac{1}{p}} \\
& \times\left(\int_{a}^{\varkappa} h_{1}^{q}(s) \varphi_{1}^{q}\left(\left(\frac{s^{\rho}-a^{\rho}}{\rho}\right)^{1-\vartheta_{1}}|\varpi(s)|\right) \frac{d s}{s^{1-\rho}}\right)^{\frac{1}{q}} \\
& \left.\left.+\frac{1}{\Gamma\left(\vartheta_{1}\right)}\left(\frac{\varkappa^{\rho}-a^{\rho}}{\rho}\right)^{1-\vartheta_{1}} \mid \frac{\varkappa^{\rho}-s^{\rho}}{\rho}\right)^{p\left(\vartheta_{1}-1\right)}\left(\frac{s^{\rho}-a^{\rho}}{\rho}\right)^{p \gamma_{2}} e^{-p \delta_{2}\left(s^{\rho}-a^{\rho}\right)} \frac{d s}{s^{1-\rho}}\right)^{\frac{1}{p}} \\
& \left.\times\left(\int_{a}^{\varkappa}\left(\frac{\varkappa^{\rho}-a^{\rho}}{\rho}\right)^{1-\left(\vartheta_{1}-\vartheta_{2}\right)}\left|\mathfrak{D}_{a}^{\vartheta_{1}} \varpi(s)\right|\right) \frac{d s}{s^{1-\rho}}\right)^{\frac{1}{q}} . \\
& \times\left(\int _ { a } ^ { \varkappa } h _ { 2 } ^ { q } ( s ) \varphi _ { 2 } ^ { q } \left(\left(\frac{s^{\prime}}{\rho}\right.\right.\right.
\end{aligned}
$$

Since $q>\frac{1}{\vartheta_{1}-\vartheta_{2}}, \gamma_{k}>\frac{1}{q}-1, \delta_{k}>0$, we have $p\left(\vartheta_{1}-1\right)+1>0$ and $1+p \gamma_{k}>0, k=1,2$, so we can apply Lemma 3.1 to get

$$
\begin{aligned}
& \left(\frac{\varkappa^{\rho}-a^{\rho}}{\rho}\right)^{1-\vartheta_{1}}|\varpi(\varkappa)| \\
& \leq K_{1}+C_{3}\left[\left(\int_{a}^{\varkappa} h_{1}^{q}(s) \varphi_{1}^{q}\left(\left(\frac{s^{\rho}-a^{\rho}}{\rho}\right)^{1-\vartheta_{1}}|\varpi(s)|\right) \frac{d s}{s^{1-\rho}}\right)^{\frac{1}{q}}\right. \\
& \left.\quad+\left(\int_{a}^{\varkappa} h_{2}^{q}(s) \varphi_{2}^{q}\left(\left(\frac{s^{\rho}-a^{\rho}}{\rho}\right)^{1-\left(\vartheta_{1}-\vartheta_{2}\right)}\left|{ }^{\rho} \mathfrak{D}_{a}^{\vartheta_{1}} \varpi(s)\right|\right) \frac{d s}{s^{1-\rho}}\right)^{\frac{1}{q}}\right] .
\end{aligned}
$$

Also we have

$$
\begin{aligned}
\rho^{\rho} \mathfrak{D}_{a}^{\vartheta_{2}} \varpi(\varkappa)= & \frac{b}{\Gamma\left(\vartheta_{1}-\vartheta_{2}\right)}\left(\frac{\varkappa^{\rho}-a^{\rho}}{\rho}\right)^{\vartheta_{1}-\vartheta_{2}-1} \\
& +\frac{1}{\Gamma\left(\vartheta_{1}-\vartheta_{2}\right)} \int_{a}^{\varkappa}\left(\frac{\varkappa^{\rho}-s^{\rho}}{\rho}\right)^{\vartheta_{1}-\vartheta_{2}-1}{ }^{\rho} \mathfrak{D}_{a}^{\vartheta_{1}} \varpi(s) \frac{d s}{s^{1-\rho}} \\
= & \frac{b}{\Gamma\left(\vartheta_{1}-\vartheta_{2}\right)}\left(\frac{\varkappa^{\rho}-a^{\rho}}{\rho}\right)^{\vartheta_{1}-\vartheta_{2}-1}+\frac{1}{\Gamma\left(\vartheta_{1}-\vartheta_{2}\right)} \\
& \times \int_{a}^{\varkappa}\left(\frac{\varkappa^{\rho}-s^{\rho}}{\rho}\right)^{\vartheta_{1}-\vartheta_{2}-1} \mathfrak{F}\left(s, \varpi(s),{ }^{\rho} \mathfrak{D}_{a}^{\vartheta_{2}} \varpi(s)\right) \frac{d s}{s^{1-\rho}} .
\end{aligned}
$$


Multiplying (3.21) by $\left(\frac{\varkappa^{\rho}-a^{\rho}}{\rho}\right)^{1-\left(\vartheta_{1}-\vartheta_{2}\right)}$ and using (3.2), we find that

$$
\begin{aligned}
& \left.\left(\frac{\varkappa^{\rho}-a^{\rho}}{\rho}\right)^{1-\left(\vartheta_{1}-\vartheta_{2}\right)}\right|^{\rho} \mathfrak{D}_{a}^{\vartheta_{2}} \varpi(\varkappa) \mid \\
& \leq K_{1}+\frac{\left(\frac{\varkappa^{\rho}-a^{\rho}}{\rho}\right)^{1-\left(\vartheta_{1}-\vartheta_{2}\right)}}{\Gamma\left(\vartheta_{1}-\vartheta_{2}\right)} \int_{a}^{\varkappa}\left(\frac{\varkappa^{\rho}-s^{\rho}}{\rho}\right)^{\vartheta_{1}-\vartheta_{2}-1}\left(\frac{s^{\rho}-a^{\rho}}{\rho}\right)^{\gamma_{1}} e^{-\delta_{1}\left(s^{\rho}-a^{\rho}\right)} \\
& \quad \times h_{1}(s) \varphi_{1}\left(\left(\frac{s^{\rho}-a^{\rho}}{\rho}\right)^{1-\vartheta_{1}}|\varpi(s)|\right) \frac{d s}{s^{1-\rho}} \\
& \quad+\frac{\left(\frac{\varkappa^{\rho}-a^{\rho}}{\rho}\right)^{1-\left(\vartheta_{1}-\vartheta_{2}\right)}}{\Gamma\left(\vartheta_{1}-\vartheta_{2}\right)} \int_{a}^{\varkappa}\left(\frac{\varkappa^{\rho}-s^{\rho}}{\rho}\right)^{\vartheta_{1}-\vartheta_{2}-1}\left(\frac{s^{\rho}-a^{\rho}}{\rho}\right)^{\gamma_{2}} e^{-\delta_{2}\left(s^{\rho}-a^{\rho}\right)} \\
& \quad \times h_{2}(s) \varphi_{2}\left(\left(\frac{s^{\rho}-a^{\rho}}{\rho}\right)^{1-\left(\vartheta_{1}-\vartheta_{2}\right)}\left|\rho \mathfrak{D}_{a}^{\vartheta_{2}} \varpi(s)\right|\right) \frac{d s}{s^{1-\rho}} .
\end{aligned}
$$

From the Hölder inequality, we have

$$
\begin{aligned}
& \left(\frac{\varkappa^{\rho}-a^{\rho}}{\rho}\right)^{1-\left(\vartheta_{1}-\vartheta_{2}\right)}\left|\mathfrak{D}_{a}^{\vartheta_{2}} \varpi(\varkappa)\right| \\
& \leq K_{1}+\frac{\left(\frac{\varkappa^{\rho}-a^{\rho}}{\rho}\right)^{1-\left(\vartheta_{1}-\vartheta_{2}\right)}}{\Gamma\left(\vartheta_{1}-\vartheta_{2}\right)} \\
& \quad \times\left(\int_{a}^{\varkappa}\left(\frac{\varkappa^{\rho}-s^{\rho}}{\rho}\right)^{p\left(\vartheta_{1}-\vartheta_{2}-1\right)}\left(\frac{s^{\rho}-a^{\rho}}{\rho}\right)^{p \gamma_{1}} e^{-p \delta_{1}\left(s^{\rho}-a^{\rho}\right)} \frac{d s}{s^{1-\rho}}\right)^{\frac{1}{p}} \\
& \quad \times\left(\int_{a}^{\varkappa} h_{1}^{q}(s) \varphi_{1}^{q}\left(\left(\frac{s^{\rho}-a^{\rho}}{\rho}\right)^{1-\vartheta_{1}}|\varpi(s)|\right) \frac{d s}{s^{1-\rho}}\right)^{\frac{1}{q}} \\
& \quad+\frac{\left(\frac{\varkappa^{\rho}-a^{\rho}}{\rho}\right)^{1-\left(\vartheta_{1}-\vartheta_{2}\right)}}{\Gamma\left(\vartheta_{1}-\vartheta_{2}\right)}\left(\int_{a}^{\varkappa}\left(\frac{\varkappa^{\rho}-s^{\rho}}{\rho}\right)^{p\left(\vartheta_{1}-\vartheta_{2}-1\right)}\left(\frac{s^{\rho}-a^{\rho}}{\rho}\right)^{p \gamma_{2}} e^{-p \delta_{2}\left(s^{\rho}-a^{\rho}\right)} \frac{d s}{s^{1-\rho}}\right)^{\frac{1}{p}} \\
& \quad \times\left(\int_{a}^{\varkappa} h_{2}^{q}(s) \varphi_{2}^{q}\left(\left(\frac{s^{\rho}-a^{\rho}}{\rho}\right)^{1-\left(\vartheta_{1}-\vartheta_{2}\right)}||^{\rho} \mathfrak{D}_{a}^{\vartheta_{2}} \varpi(s) \mid\right) \frac{d s}{s^{1-\rho}}\right)^{\frac{1}{q}} .
\end{aligned}
$$

Applying Lemma 3.1, we obtain

$$
\begin{aligned}
& \left(\frac{\varkappa^{\rho}-a^{\rho}}{\rho}\right)^{1-\left(\vartheta_{1}-\vartheta_{2}\right)}\left|{ }^{\rho} \mathfrak{D}_{a}^{\vartheta_{2}} \varpi(\varkappa)\right| \\
& \leq K_{1}+C_{3}^{\prime}\left[\left(\int_{a}^{\varkappa} h_{1}^{q}(s) \varphi_{1}^{q}\left(\left(\frac{s^{\rho}-a^{\rho}}{\rho}\right)^{1-\vartheta_{1}}|\varpi(s)|\right) \frac{d s}{s^{1-\rho}}\right)^{\frac{1}{q}}\right. \\
& \left.\quad+\left(\int_{a}^{\varkappa} h_{2}^{q}(s) \varphi_{2}^{q}\left(\left(\frac{s^{\rho}-a^{\rho}}{\rho}\right)^{1-\left(\vartheta_{1}-\vartheta_{2}\right)}\left|{ }^{\rho} \mathfrak{D}_{a}^{\vartheta_{2}} \varpi(s)\right|\right) \frac{d s}{s^{1-\rho}}\right)^{\frac{1}{q}}\right] .
\end{aligned}
$$

Equation (3.17) is an immediate consequence of (3.18), (3.20) and (3.22).

Theorem 3.4 Assume that the assumptions of Lemma 3.2 hold, then the solutions of (1.1) satisfy

$$
|\varpi(\varkappa)| \leq C\left(\frac{\varkappa^{\rho}-a^{\rho}}{\rho}\right)^{\vartheta_{1-1}} \text { and }
$$


Kassim et al. Advances in Difference Equations

(2021) $2021: 376$

Page 14 of 20

$$
\left|{ }^{\rho} \mathfrak{D}_{a}^{\vartheta_{2}} \varpi(\varkappa)\right|<C\left(\frac{\varkappa^{\rho}-a^{\rho}}{\rho}\right)^{\vartheta_{1}-\vartheta_{2}-1}, \quad C>0, \varkappa>a
$$

provided that

$$
\int_{\varkappa_{0}}^{\infty} \frac{d s}{\varphi_{1}^{q}\left(s^{\frac{1}{q}}\right) \varphi_{2}^{q}\left(s^{\frac{1}{q}}\right)}=\infty, \quad \varkappa_{0}>0
$$

Proof Thanks to Lemma 3.2 and the fact that $\varphi_{k}, k=1,2$, are nondecreasing, we have

$$
\left\{\begin{array}{l}
\varphi_{1}\left[\left(\frac{\varkappa^{\rho}-a^{\rho}}{\rho}\right)^{1-\vartheta_{1}}|\varpi(\varkappa)|\right] \leq \varphi_{1}[z(\varkappa)], \quad \varkappa>a \\
\varphi_{2}\left[\left.\left(\frac{\varkappa^{\rho}-a^{\rho}}{\rho}\right)^{1-\left(\vartheta_{1}-\vartheta_{2}\right)}\right|^{\rho} \mathfrak{D}_{a}^{\vartheta_{2}} \varpi(\varkappa) \mid\right] \leq \varphi_{2}[z(\varkappa)], \quad \varkappa>a .
\end{array}\right.
$$

Therefore (3.8) and (3.23), lead to

$$
z(\varkappa) \leq K_{1}+K_{2}\left(\int_{a}^{\varkappa} h^{q}(s) \varphi_{1}^{q}(z(s)) \varphi_{2}^{q}(z(s)) d s\right)^{\frac{1}{q}}, \quad \varkappa>a .
$$

Applying Lemma 2.10 to (3.24), we get

$$
z^{q}(\varkappa) \leq B_{1}+B_{2} \int_{a}^{\varkappa} h^{q}(s) \varphi_{1}^{q}(z(s)) \varphi_{2}^{q}(z(s)) d s, \quad \varkappa>a,
$$

where

$$
B_{1}=2^{q-1} K_{1} \quad \text { and } \quad B_{2}=2^{q-1} K_{2} .
$$

Now, put $u(\varkappa)=z^{q}(\varkappa)$, then (3.25) becomes

$$
u(\varkappa) \leq B_{1}+B_{2} \int_{a}^{\varkappa} h^{q}(s) \varphi_{1}^{q}\left(u^{\frac{1}{q}}(s)\right) \varphi_{2}^{q}\left(u^{\frac{1}{q}}(s)\right) d s, \quad \varkappa>a .
$$

Let

$$
w(r)=\varphi_{1}^{q}\left(r^{\frac{1}{q}}\right) \varphi_{2}^{q}\left(r^{\frac{1}{q}}\right) .
$$

Then $w$ is a nondecreasing continuous function and

$$
u(\varkappa) \leq B_{1}+B_{2} \int_{a}^{\varkappa} h^{q}(s) w(u) d s, \quad \varkappa>a .
$$

Applying Theorem 2.9 to (3.28), we obtain

$$
u(\varkappa) \leq G^{-1}\left(G\left(B_{1}\right)+B_{2} \int_{a}^{\varkappa} h^{q}(s) d s\right), \quad \varkappa>a,
$$

where

$$
G(\varkappa)=\int_{\varkappa_{0}}^{\varkappa} \frac{d s}{w(s)}=\int_{\varkappa_{0}}^{\varkappa} \frac{d s}{\varphi_{1}^{q}\left(s^{\frac{1}{q}}\right) \varphi_{2}^{q}\left(s^{\frac{1}{q}}\right)}, \quad \varkappa_{0}>0, \varkappa>0 .
$$


Clearly

$$
G\left(B_{1}\right)+B_{2} \int_{a}^{\varkappa} h^{q}(s) d s \in \operatorname{Dom}\left(G^{-1}\right), \varkappa>a .
$$

As $h \in L_{q} \in(a, \infty)$, we have

$$
H_{1}=G\left(B_{1}\right)+B_{2} \int_{a}^{\infty} h^{q}(s) d s<\infty
$$

and

$$
u(\varkappa) \leq H_{2}:=G^{-1}\left(H_{1}\right)<\infty .
$$

Therefore $z(\varkappa) \leq C:=H_{2}^{\frac{1}{q}}$ and the result follows.

Theorem 3.5 Assume that the assumptions of Lemma 3.3 hold, then the solutions of (1.1) satisfy

$$
\begin{aligned}
& |\varpi(\varkappa)| \leq C\left(\frac{\varkappa^{\rho}-a^{\rho}}{\rho}\right)^{\vartheta_{1}-1} \text { and } \\
& \left|\mathfrak{D}_{a}^{\vartheta_{2}} \varpi(\varkappa)\right|<C\left(\frac{\varkappa^{\rho}-a^{\rho}}{\rho}\right)^{\vartheta_{1}-\vartheta_{2}-1}, \quad C>0, \varkappa>a,
\end{aligned}
$$

provided that

$$
\int_{\varkappa_{0}}^{\infty} \frac{d s}{\varphi_{1}^{q}\left(s^{\frac{1}{q}}\right)+\varphi_{2}^{q}\left(s^{\frac{1}{q}}\right)}=\infty, \quad \varkappa_{0}>0
$$

Proof By using Lemma 3.3, we deduce

$$
\begin{aligned}
& \varphi_{1}\left(\left(\frac{\varkappa^{\rho}-a^{\rho}}{\rho}\right)^{1-\vartheta_{1}}|\varpi(\varkappa)|\right) \leq \varphi_{1}(z(\varkappa)), \quad \varkappa>a, \\
& \varphi_{2}\left(\left(\frac{\varkappa^{\rho}-a^{\rho}}{\rho}\right)^{1-\left(\vartheta_{1}-\vartheta_{2}\right)}||^{\rho} \mathfrak{D}_{a}^{\vartheta_{2}} \varpi(\varkappa) \mid\right) \leq \varphi_{2}(z(\varkappa)), \quad \varkappa>a,
\end{aligned}
$$

where $z(\varkappa)$ is as in (3.18). Take into consideration (3.18) and (3.31) we find that

$$
z(\varkappa) \leq K_{1}+K_{2}\left[\left(\int_{a}^{\varkappa} h_{1}^{q}(s) \varphi_{1}^{q}(z(s)) d s\right)^{\frac{1}{q}}+\left(\int_{a}^{\varkappa} h_{2}^{q}(s) \varphi_{2}^{q}(z(s)) d s\right)^{\frac{1}{q}}\right] .
$$

By using Lemma (2.10), we have

$$
z^{q}(\varkappa) \leq B_{1}+B_{2}\left[\int_{a}^{\varkappa} h_{1}^{q}(s) \varphi_{1}^{q}(z(s)) d s+\int_{a}^{\varkappa} h_{2}^{q}(s) \varphi_{2}^{q}(z(s)) d s\right],
$$

where

$$
B_{1}=2^{q-1} K_{1}^{q} \quad \text { and } \quad B_{2}=2^{2(q-1)} K_{2}^{q} \text {. }
$$


Furthermore, due to the inequality

$$
h_{1}^{q}(s) \varphi_{1}^{q}(z(s))+h_{2}^{q}(s) \varphi_{2}^{q}(z(s)) \leq\left[h_{1}^{q}(s)+h_{2}^{q}(s)\right]\left[\varphi_{1}^{q}(z(s))+\varphi_{2}^{q}(z(s))\right]
$$

we have by (3.33) and (3.34)

$$
z^{q}(\varkappa) \leq B_{1}+B_{2} \int_{a}^{\varkappa}\left[h_{1}^{q}(s)+h_{2}^{q}(s)\right]\left[\varphi_{1}^{q}(z(s))+\varphi_{2}^{q}(z(s))\right] d s .
$$

Now, let $u(\varkappa)=z^{q}(\varkappa)$. Then (3.35) becomes

$$
u(\varkappa) \leq B_{1}+B_{2} \int_{a}^{\varkappa}\left[h_{1}^{q}(s)+h_{2}^{q}(s)\right]\left[\varphi_{1}^{q}\left(u^{\frac{1}{q}}(s)\right)+\varphi_{2}^{q}\left(u^{\frac{1}{q}}(s)\right)\right] d s, \quad \varkappa>a .
$$

Let

$$
g(r)=\varphi_{1}^{q}\left(r^{\frac{1}{q}}\right)+\varphi_{2}^{q}\left(r^{\frac{1}{q}}\right) .
$$

Then $g$ is nondecreasing continuous function, since $\varphi_{1}$ and $\varphi_{2}$ are nondecreasing continuous functions.

Hence, from (3.36) and (3.37), we get

$$
u(\varkappa) \leq B_{1}+B_{2} \int_{a}^{\varkappa}\left[h_{1}^{q}(s)+h_{2}^{q}(s)\right] g(u(s)) d s, \quad \varkappa>a .
$$

Applying Theorem 2.9 to (3.38), we have

$$
u(\varkappa) \leq G^{-1}\left(G\left(B_{1}\right)+B_{2} \int_{a}^{\varkappa}\left[h_{1}^{q}(s)+h_{2}^{q}(s)\right] d s\right), \quad \varkappa>a,
$$

where

$$
G(\varkappa)=\int_{\varkappa_{0}}^{\varkappa} \frac{d s}{g(s)}=\int_{\varkappa_{0}}^{\varkappa} \frac{d s}{\varphi_{1}^{q}\left(s^{\frac{1}{q}}\right)+\varphi_{2}^{q}\left(s^{\frac{1}{q}}\right)}, \quad \varkappa_{0}>0, \varkappa>0 .
$$

As $h_{k} \in L_{q} \in(a, \infty)$, we let

$$
H_{1}=G\left(B_{1}\right)+B_{2} \int_{a}^{\infty}\left[h_{1}^{q}(s)+h_{2}^{q}(s)\right] d s
$$

Therefore

$$
u(\varkappa) \leq H_{2}:=G^{-1}\left(H_{1}\right)<\infty
$$

Next, $u(\varkappa)=z^{q}(\varkappa)$ implies that $z(\varkappa) \leq C:=H_{2}^{\frac{1}{q}}$. Then we get from (3.17)

$$
|\varpi(\varkappa)| \leq C\left(\frac{\varkappa^{\rho}-a^{\rho}}{\rho}\right)^{\vartheta_{1}-1} \text { and }\left|\rho \mathfrak{D}_{a}^{\vartheta_{2}} \varpi(\varkappa)\right|<C\left(\frac{\varkappa^{\rho}-a^{\rho}}{\rho}\right)^{\vartheta_{1}-\vartheta_{2}-1}, \quad \varkappa>a \text {. }
$$


Kassim et al. Advances in Difference Equations

(2021) 2021:376

Page 17 of 20

4 Examples

Example 4.1 Consider the problem

$$
\left\{\begin{aligned}
{ }^{\rho} \mathfrak{D}_{a}^{1 / 2} \varpi(\varkappa)= & \left(\frac{\varkappa^{\rho}-a^{\rho}}{\rho}\right)^{3}\left(\cos \varpi^{2}\right)\left(\sin \varkappa^{\rho-1}\right) \\
& \times e^{-2\left(\varkappa^{\rho}-a^{\rho}\right)}[\varpi(\varkappa)]^{1 / 4}\left[{ }^{\rho} \mathfrak{D}_{a}^{1 / 3} \varpi(\varkappa)\right]^{1 / 5}, \quad \varkappa>a, \\
\rho & \left.\mathfrak{I}_{a}^{1 / 2} \varpi(\varkappa)\right|_{\varkappa=a}=b .
\end{aligned}\right.
$$

Here we have

$$
\begin{aligned}
\mid \mathfrak{F} & \left(\varkappa, \varpi(\varkappa),{ }^{\rho} \mathfrak{D}_{a}^{1 / 4} \varpi(\varkappa)\right) \mid \\
= & \left|\left(\frac{\varkappa^{\rho}-a^{\rho}}{\rho}\right)^{3}\left(\cos \varpi^{2}\right)\left(\sin \varkappa^{\rho-1}\right) e^{-2\left(\varkappa^{\rho}-a^{\rho}\right)}[\varpi(\varkappa)]^{1 / 4}\left[{ }^{\rho} \mathfrak{D}_{a}^{1 / 3} \varpi(\varkappa)\right]^{1 / 5}\right| \\
\leq & \mid \varkappa^{\rho-1}\left(\frac{\varkappa^{\rho}-a^{\rho}}{\rho}\right)^{3-1 / 8-1 / 6} e^{-2\left(\varkappa^{\rho}-a^{\rho}\right)} \\
& \times\left[\left(\frac{\varkappa^{\rho}-a^{\rho}}{\rho}\right)^{1 / 2} \varpi(\varkappa)\right]^{1 / 4}\left[\left(\frac{\varkappa^{\rho}-a^{\rho}}{\rho}\right)^{5 / 6}{ }^{\rho} \mathfrak{D}_{a}^{1 / 3} \varpi(\varkappa)\right]^{1 / 5} \mid \\
\leq & \left(\frac{\varkappa^{\rho}-a^{\rho}}{\rho}\right)^{\gamma} e^{-\left(\varkappa^{\rho}-a^{\rho}\right)} h(\varkappa) \varphi_{1}\left[\left(\frac{\varkappa^{\rho}-a^{\rho}}{\rho}\right)^{1-1 / 2} \varpi(\varkappa)\right] \\
& \times \varphi_{2}\left[\left(\frac{\varkappa^{\rho}-a^{\rho}}{\rho}\right)^{1-(1 / 2-1 / 3)} \mathfrak{D}_{a}^{1 / 3} \varpi(\varkappa)\right],
\end{aligned}
$$

where $\gamma=\frac{17}{24}, h(\varkappa)=\varkappa^{\rho-1}\left(\frac{\varkappa^{\rho}-a^{\rho}}{\rho}\right)^{2} e^{-\left(\varkappa^{\rho}-a^{\rho}\right)}, \varphi_{1}(\varkappa)=\varkappa^{1 / 4}$ and $\varphi_{2}(\varkappa)=\varkappa^{1 / 5}$. All the condiions of Theorem 3.4 are satisfied. Then

$$
|\varpi(\varkappa)| \leq C\left(\frac{\varkappa^{\rho}-a^{\rho}}{\rho}\right)^{-1 / 2} \text { and }\left|{ }^{\rho} \mathfrak{D}_{a}^{1 / 3} \varpi(\varkappa)\right| \leq C\left(\frac{\varkappa^{\rho}-a^{\rho}}{\rho}\right)^{-5 / 6}, \quad \varkappa>a .
$$

Example 4.2 Consider the problem

$$
\left\{\begin{aligned}
& \begin{array}{rl}
\mathfrak{D}_{a}^{1 / 3} \varpi(\varkappa)= & \left(\frac{\varkappa^{\rho}-a^{\rho}}{\rho}\right)^{2}\left(\cos \varpi^{2}\right) e^{-2\left(\varkappa^{\rho}-a^{\rho}\right)-3 \varkappa}[\varpi(\varkappa)]^{1 / 2} \\
& +\left(\frac{\varkappa^{\rho}-a^{\rho}}{\rho}\right)^{2} \varkappa^{-2} e^{-3\left(\varkappa^{\rho}-a^{\rho}\right)}\left(\cos \varkappa^{2}\right)\left[{ }^{\rho} \mathfrak{D}_{a}^{1 / 4} \varpi(\varkappa)\right]^{1 / 2}, \quad \varkappa>a,
\end{array} \\
& \left.\rho \mathfrak{I}_{a}^{2 / 3} \varpi(\varkappa)\right|_{\varkappa=a}=b .
\end{aligned}\right.
$$

We can rewrite the right hand side of (4.2) as follows:

$$
\begin{aligned}
& \mid\left(\frac{\varkappa^{\rho}-a^{\rho}}{\rho}\right)^{2}\left(\cos \varpi^{2}\right) e^{-2\left(\varkappa^{\rho}-a^{\rho}\right)} e^{-3 \varkappa}[\varpi(\varkappa)]^{1 / 2} \\
& \quad+\left(\frac{\varkappa^{\rho}-a^{\rho}}{\rho}\right)^{2} e^{-3\left(\varkappa^{\rho}-a^{\rho}\right)}\left(\cos \varkappa^{2}\right) \varkappa^{-2}\left[{ }^{\rho} \mathfrak{D}_{a}^{1 / 4} \varpi(\varkappa)\right]^{1 / 2} \mid \\
& \leq\left(\frac{\varkappa^{\rho}-a^{\rho}}{\rho}\right)^{\gamma_{1}} e^{-2\left(\varkappa^{\rho}-a^{\rho}\right)} h_{1}(\varkappa) \varphi_{1}\left[\left(\frac{\varkappa^{\rho}-a^{\rho}}{\rho}\right)^{1-1 / 3} \varpi(\varkappa)\right] \\
& \quad+\left(\frac{\varkappa^{\rho}-a^{\rho}}{\rho}\right)^{\gamma_{2}} e^{-3\left(\varkappa^{\rho}-a^{\rho}\right)} h_{2}(\varkappa) \varphi_{2}\left[\left(\frac{\varkappa^{\rho}-a^{\rho}}{\rho}\right)^{1-(1 / 3-1 / 4)} \rho \mathfrak{D}_{a}^{1 / 4} \varpi(\varkappa)\right],
\end{aligned}
$$


where $\gamma_{1}=8 / 3, \gamma_{2}=37 / 24, h_{1}(\varkappa)=e^{-3 \varkappa}, h_{2}(\varkappa)=\varkappa^{-2}$ and $\varphi_{1}(\varkappa)=\varphi_{2}(\varkappa)=\varkappa^{1 / 2}$. Obviously, all conditions of Theorem 3.5 are satisfied. Then

$$
\begin{aligned}
& |\varpi(\varkappa)| \leq C\left(\frac{\varkappa^{\rho}-a^{\rho}}{\rho}\right)^{-1 / 2} \text { and } \\
& \left|{ }^{\rho} \mathfrak{D}_{a}^{1 / 4} \varpi(\varkappa)\right| \leq C\left(\frac{\varkappa^{\rho}-a^{\rho}}{\rho}\right)^{-3 / 4}, \quad \varkappa>a .
\end{aligned}
$$

\section{Remark 4.3}

(i) The reported fractional operator here generalizes both the Riemann-Liouville and Hadamard fractional operators in one form, and it is also most regarding the Erdélyi-Kober fractional operator, especially, when $\rho \rightarrow 1$, we get a Riemann-Liouville fractional derivative and doing $\rho \downarrow 0$, we get a Caputo-Hadamard fractional derivative. Also, we get (Liouville and Weyl) for $\rho \rightarrow 1,(a=0$ and $a=-\infty)$, respectively.

(ii) The results obtained in this work will remain valid if we take into account the aforementioned special cases.

(iii) Our current problem (1.1) provides a general platform that covers most of the classic problems from (1.2) into (1.8) mentioned in the introduction Sect.

\section{Concluding remarks}

In the present work, we have investigated the stability of solutions for some fractional differential problems. These types of equations involved the generalized fractional derivative of different orders. In fact, we have established sufficient conditions on the nonlinear terms, via making use of some modified generalized versions of inequalities, to study the decay of solutions to zero in terms of a power function. Besides, some characteristics of the generalized fractional derivative and appropriate regularization techniques have been employed. Ultimately, this paper concludes with relevant examples to confirm the legitimacy of the acquired results.

In future work, many cases can be established when one takes a more generalised operator that contains another function instead of $\varkappa^{\rho}$ in the structure of its kernel. For instance, the generalized Caputo [37] (or Hilfer [38]) fractional operators. Further, it will be of interest to study the existing problem in this article for the Mittag-Leffler power law [39] and for fractal fractional operators [40].

\section{Acknowledgements}

The authors want to thank the anonymous reviewers sincerely for their valuable comments to improve paper quality.

\section{Funding}

Not applicable.

Availability of data and materials

Not applicable in this study.

Competing interests

The authors declare that they have no competing interests. 


\section{Author details}

'Department of Basic Engineering Sciences, College of Engineering, Imam Abdulrahman Bin Faisal University, Dammam 34151, Saudi Arabia. '2Department of Mathematics and General Sciences, Prince Sultan University, Riyadh, Saudi Arabia. ${ }^{3}$ Department of Medical Research, China Medical University, Taichung 40402, Taiwan. ${ }^{4}$ Department of Computer Science and Information Engineering, Asia University, Taichung, Taiwan. ${ }^{5}$ Department of Mathematics, Hodeidah University, Al-Hodeidah, Yemen.

\section{Publisher's Note}

Springer Nature remains neutral with regard to jurisdictional claims in published maps and institutional affiliations.

Received: 22 April 2021 Accepted: 28 July 2021 Published online: 11 August 2021

\section{References}

1. Bagley, R.L., Torvik, P.J.: A theoretical basis for the application of fractional calculus to viscoelasticity. J. Rheol. 27 201-210 (1983)

2. Bagley, R.L., Torvik, P.J.: A different approach to the analysis of viscoelastically damped structures. AIAA J. 21, 741-748 (1983)

3. Bagley, R.L., Torvik, P.J.: On the appearance of the fractional derivative in the behavior of real material. J. Appl. Mech. 51, 294-298 (1983)

4. Kilbas, A.A., Srivastava, H.M., Trujillo, J.J.: Theory and Applications of Fractional Differential Equations. North-Holland Mathematics Studies, vol. 204. Elsevier, Amsterdam (2009)

5. Katugampola, U.N.: A new approach to generalized fractional derivatives. Bull. Math. Anal. Appl. 6, 1-15 (2014)

6. Almeida, R., Malinowska, A.B., Odzijewicz, T.: Fractional differential equations with dependence on the Caputo-Katugampola derivative (2016) arXiv:1607.06913

7. Redhwan, S.S., Shaikh, S.L., Abdo, M.S.: Implicit fractional differential equation with anti-periodic boundary condition involving Caputo-Katugampola type. AIMS Math. 5(4), 3714-3730 (2020)

8. Abdo, M.S., Thabet, S.T., Ahmad, B.: The existence and Ulam-Hyers stability results for $\psi$-Hilfer fractional integrodifferential equations. J. Pseudo-Differ. Oper. Appl. 11(4), 1757-1780 (2020) https://doi.org/10.1007/s11868-020-00355-x

9. Abdo, M.S.: Further results on the existence of solutions for generalized fractional quadratic functional integral equations. J. Math. Anal. Model. 1(1), 33-46 (2020). https://doi.org/10.48185/jmam.v1i1.2

10. Redhwan, S.S., Shaikh, S.L., Abdo, M.S.: Theory of nonlinear Caputo-Katugampola fractional differential equations (2019) arXiv preprint. arXiv:1911.08884

11. Almalahi, M.A., Abdo, M.S., Panchal, S.K.: On the theory of fractional terminal value problem with $\psi$-Hilfer fractional derivative. AIMS Math. 5(5), 4889-4908 (2020)

12. Oliveira, D.S., Oliveira, E.C.: Hilfer-Katugampola fractional derivatives. Comput. Appl. Math. 37, 3672-3690 (2018)

13. Benchohra, M., Bouriah, S., Nieto, J.J.: Terminal value problem for differential equations with Hilfer-Katugampola fractional derivative. Symmetry 11(5), 672 (2019). https://doi.org/10.3390/sym11050672

14. Abdo, M.S., Abdeljawad, T., Kucche, K.D., Alqudah, M.A., Ali, S.M., Jeelani, M.B.: On nonlinear pantograph fractional differential equations with Atangana-Baleanu-Caputo derivative. Adv. Differ. Equ. 2021(1), 1 (2021)

15. Abdo, M.S., Abdeljawad, T., Ali, S.M., Shah, K.: On fractional boundary value problems involving fractional derivatives with Mittag-Leffler kernel and nonlinear integral conditions. Adv. Differ. Equ. 2021(1), 1 (2021)

16. Abbas, S.: Existence of solutions to fractional order ordinary and delay differential equations and applications. Electron. J. Differ. Equ. 2011, 9 (2011)

17. Abbas, S.: Weighted pseudo almost automorphic solutions of fractional functional differential equations. CUBO 16(1) 21-36 (2014)

18. Kavitha, V., Abbas, S., Murugesu, R.: Asymptotically almost automorphic solutions of fractional order neutral integro-differential equations. Bull. Malays. Math. Sci. Soc. 39(3), 1075-1088 (2016)

19. Furati, K.M., Tatar, N.-E.: Power type estimates for a nonlinear fractional differential equation. Nonlinear Anal. TMA 62 1025-1036 (2005)

20. Furati, K.M., Kassim, M.D., Tatar, N.-E.: Existence and uniqueness for a problem involving Hilfer fractional derivative. Comput. Math. Appl. 64, 1616-1626 (2012)

21. Płociniczak, Ł.: On asymptotics of some fractional differential equations. Math. Model. Anal. 18(3), 358-373 (2013)

22. Medved, M., Pospišsil, M.: Asymptotic integration of fractional differential equations with integrodifferential right-hand side. Math. Model. Anal. 20(4), 471-489 (2015)

23. Kassim, M.D., Furati, K.M., Tatar, N.-E.: Asymptotic behavior of solutions to nonlinear initial-value fractional differential problems. Electron. J. Differ. Equ. 291, 1 (2016)

24. Kassim, M.D., Tatar, N.-E.: Stability of logarithmic type for a Hadamard fractional differential problem. J. Pseudo-Differ Oper. Appl. 11, 447-466 (2020)

25. Kassim, M.D., Tatar, N.-E.: Convergence of solutions of fractional differential equations to power-type functions. Electron. J. Differ. Equ. 2020, 111 (2020)

26. Medved, M.: On the asymptotic behavior of solutions of nonlinear differential equations of integer and also of non-integer order. Electron. J. Qual. Th. Diff. Eq., Proc. 9th Coll. QTDE. 10, 1-9 (2012)

27. Medved, M.: Asymptotic integration of some classes of fractional differential equations. Tatra Mt. Math. Publ. 54 $119-132(2013)$

28. Kassim, M.D., Furati, K.M., Tatar, N.-E.: Asymptotic behavior of solutions to nonlinear fractional differential equations. Math. Model. Anal. 21, 610-629 (2016)

29. Kassim, M.D., Furati, K.M., Tatar, N.-E.: On a differential equation involving Hilfer-Hadamard fractional derivative. Abstr. Appl. Anal. 2012, Article ID 391062 (2012)

30. Kassim, M.D. Tatar, N.-E.: Well-posedness and stability for a differential problem with Hilfer-Hadamard fractional derivative. Abstr. Appl. Anal. 2013, Article ID 605029 (2013) 
31. Furati, K.M., Tatar, N.-E.: Behavior of solutions for a weighted Cauchy-type fractional differential problem. J. Fract. Calc. 28, 23-42 (2005)

32. Furati, K.M., Tatar, N.-E.: Long time behaviour for a nonlinear fractional model. J. Math. Anal. Appl. 332, 441-454 (2007)

33. Katugampola, U.A.: New approach to a generalized fractional integral. Appl. Math. Comput. 218(3), 860-865 (2011)

34. Pachpatte, B.G.: Inequalities for Differential and Integral Equations. Mathematics in Science and Engineering, vol. 197. Acad. Press, San Diego-London. (1997)

35. Anastassiou, G.A.: Opial type inequalities involving Riemann-Liouville fractional derivatives of two functions with applications. Math. Comput. Model. 48(3-4), 344-374 (2008)

36. Kuczma, M.: An Introduction to the Theory of Functional Equations and Inequalities: Cauchy's Equation and Jensen's Inequality. Birkhäuser, Basel (2009)

37. Almeida, R.: A Caputo fractional derivative of a function with respect to another function. Commun. Nonlinear Sci. Numer. Simul. 44, 460-481 (2017)

38. Sousa, J.V.C., Oliveira, C.E.: On the $\psi$-Hilfer fractional derivative. Commun. Nonlinear Sci. Numer. Simul. 60, 72-91 (2018)

39. Atangana, A., Baleanu, D.: New fractional derivatives with non-local and non-singular kernel: theory and application to heat transfer. Model. Therm. Sci. 20(2), 763-769 (2016)

40. Atangana, A.: Fractal-fractional differentiation and integration: connecting fractal calculus and fractional calculus to predict complex system. Chaos Solitons Fractals 102, 396-406 (2017)

\section{Submit your manuscript to a SpringerOpen ${ }^{\circ}$ journal and benefit from:}

- Convenient online submission

- Rigorous peer review

- Open access: articles freely available online

- High visibility within the field

- Retaining the copyright to your article

Submit your next manuscript at $\boldsymbol{~ s p r i n g e r o p e n . c o m ~}$ 